\title{
The Mobility Topography Model for Substantializing and Projecting Transportation in Cities
}

\author{
Zachary Trattner, Angelos Chronis, Angel Muñoz \\ Institute for Advanced Architecture of Catalonia \\ Barcelona, Spain \\ zacharytrattner@iaac.net, angelos.chronis@iaac.net, angel@iaac.net
}

\begin{abstract}
Cities are built on transportation strategies. The constructed urban environment is influenced by the accumulation of individual transport choices, and also by the decisions of transportation planners who continue to rely on tools designed to hasten the expansion of car culture. Past experiences incorporating cars, trains, and horses have demonstrated that paradigm shifts in mobility technology impact activity patterns in cities. Autonomous vehicles will certainly revolutionize urban mobility, but the positive and negative consequences of driverless personal transport on cities and societies remain unknown. The probability of a beneficial impact will increase with more and better tools available for analyzing existing transportation habits and projecting future possibilities. The mobility topography model (MTM) introduced in this paper is a multimodal route simulator that evaluates probable modal choice for hypothetical travelers, derived using actual ridership data, transportation infrastructure, and economic conditions. An application has been developed for users to adjust input parameters and quickly project the potential impacts of evolving technologies and changing circumstances. The MTM is flexible, general, and well suited for casting holistic projections of future scenarios, rather than for precise accounting of specific interventions. The model is demonstrated in Singapore today and into the future.
\end{abstract}

\section{Author Keywords}

Transportation; mobility; probability model; autonomous vehicles, urban planning.

\section{ACM Classification Keywords \\ I.6.1 SIMULATION AND MODELING (Model \\ Development).}

\section{INTRODUCTION}

The widespread acceptance of the car in the early $20^{\text {th }}$ century was a paradigm shift in mobility technology. However, cities around the world are experiencing a multitude of problems from excessive car use, including urban sprawl, global warming, traffic congestion, poor health, and social isolation (Litman 2002). Therefore, it is reasonable to state that the shift to cars was mishandled by past generations of planners, and the same mistakes are being repeated in developing counties today. Meanwhile, many prominent planners acknowledge that when priority was first assigned to the movement of cars over pedestrians, car culture built momentum, inaugurating the development of a two-tier transportation system that persists today.

Recent advances in autonomous vehicles (AVs) suggest that in a few short years, roads will be populated by selfdriving cars. Experience tells us that the response of planners to this emerging technology will define the character of our cities and societies for generations. A potential problem is that the main computational tool that transportation planners rely on to predict future use is still the traditional four-step traffic demand model that was developed in Detroit during the 1950s. The four-step model applied the concepts of supply and demand to transportation engineering (concepts that were pioneered in economic theory), and has been used to design roads and mass transit networks based on the needs of vehicles ever since. Without doubt, we will need new, more dynamic tools to develop mobility solutions that are appropriate for cultivating healthy and sustainable cities.

This paper presents the mobility topography model (MTM), a variation on traditional transportation modeling techniques that is designed to give planners insight into the broader effects of technological, infrastructural, and economic developments. The MTM was constructed around the example of Singapore, but the tools and procedures demonstrated here can be applied to any urban environment. Calculations for the first three steps (environment description, trip generation, and route assignment) are conducted in the Grasshopper3D plugin for Rhinoceros3D using a combination of native components and custom Python scripts. The subsequent steps (calibration, mode choice, and topographic visualization) are implemented in Processing as a standalone application with an intuitive, user-friendly interface. This approach is designed to substantialize the invisible mobility layer of cities, so that planners and politicians can intuitively process visual information as opposed to abstract figures. Applying this computational tool to the difficult work of interpreting the probability landscape that emerges from urban transportation systems, the human imagination can pursue innovative solutions to complex mobility scenarios.

\section{BACKGROUND}

Transportation planners currently have access to several advanced computational techniques for modeling human 
mobility, including agent-based models (ABMs) and neural networks. Chen [2] provides a detailed survey of many possible applications for ABMs in architecture, urban, and transportation planning. Agent-based transportation models usually simulate the actual use patterns of individual travelers and vehicles; for example, the model constructed by Aschwanden [1] included digitized representations of street lanes, buildings, and bus stops. The TRANSIMS project is a flexible and comprehensive $\mathrm{ABM}$ tool that has found many wide applications including modeling vehicular traffic, pedestrian flows, and emergency egress [13]. However, ABMs are best suited for modeling situations that occur over small time scales and in limited spatial areas, because they depend on repetitively updating the microscale decisions of individual automata. Trained neural networks are another advanced computational tool with potential applications in urban planning, such as predicting patterns of urban sprawl [6]. However, the traditional fourstep model is the most commonly used tool for planning road capacity, validating public transit demand, optimizing tolls, and more. In essence, the four-step model interprets a city as a network of links and nodes, on which trips are distributed according to demand, and then redistributed based on link capacity until equilibrium is achieved.

When implementing a transportation macro-model, it is common practice to combine household data with distance decay methods or other techniques to generate trips [4]. However, Singapore hasn't published place-of-work data, so this example MTM implementation determines trip rates using alternative methods. To arrive at reasonably accurate trip rates for employed residents, distances are measured from point locations for trip generating occupancies including employment centers, malls, hospitals, airports, etc., and are modified by accessibility indices based on distance from the centroid of the starting zone (Figure 1). Zhou, Kockelman, and Lemp [25] demonstrate the use of an accessibility index to modify trip rates, a feature that is becoming progressively more common in four-step model applications. To limit the potential for extreme results, each trip start point has multiple end points.

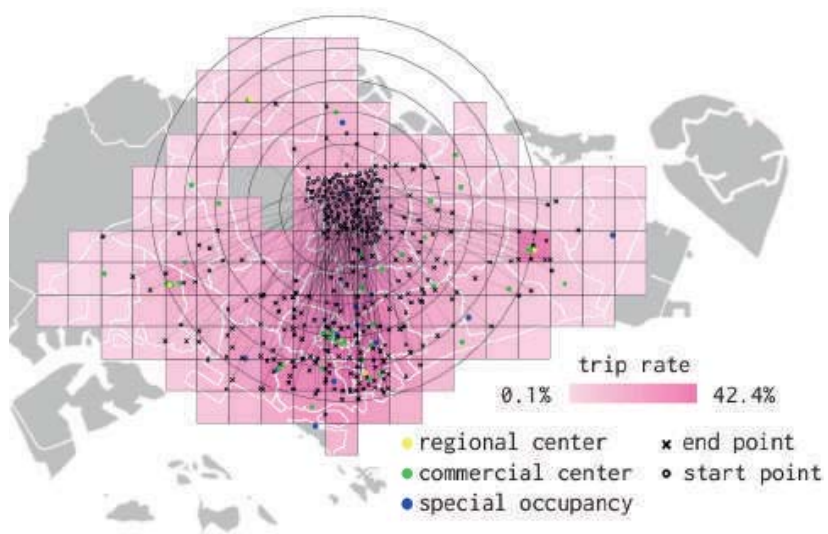

Figure 1. Employment activity trip production map for Ang Mo Kio, a centrally located, residential planning zone.
Four-step models frequently include mode choice probability calculations using probit, logit, or gravity models. The multinomial logit model (MNL) used by the MTM was adapted from the methods used by Koppelman and Bhat [12] to compare discrete utility values for several modes of transportation and determine the most probable choice (Figure 2). The utility value equation includes average monthly household income for each zone as a denominator below calculated trip cost (Figure 3), enabling the model to consider the influence of economic forces like income inequality on mobility. These formulae are well established, and the theoretical basis for the validity of the MTM is derived from the use of these models.

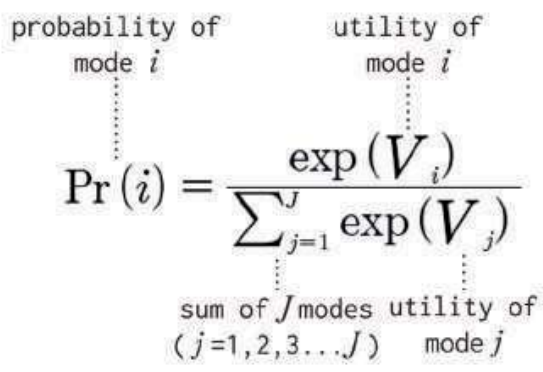

Figure 2. Probability equation derived from MNL.

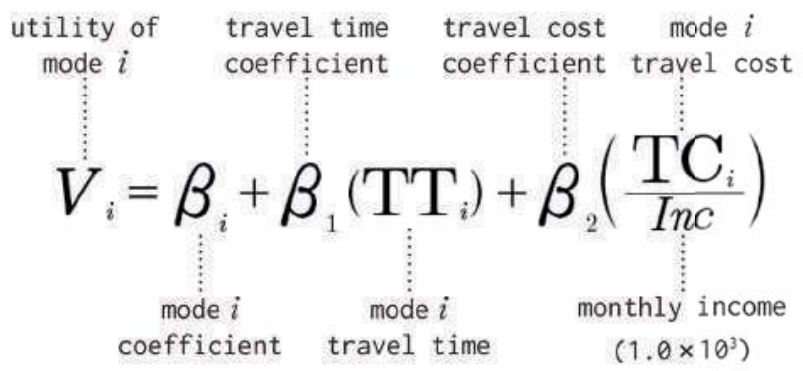

Figure 3. Equation to calculate mode utility using terms for travel time, travel cost, and income alongside mode coefficients.

The resulting probability output by the MNL is analogous to probable modal choice, and is calculated by comparing the utility of different modes. Each mode utility value is calibrated to better represent reality using mode bias coefficients. While logit models have often relied on traditional optimization techniques like Newton-Raphson or steepest ascent methods to determine mode bias coefficients, the proposed MTM uses a genetic algorithm (GA) to determine coefficients. Research confirms the viability of calibrating coefficients using GAs to match a dataset [24]. In this case, the dataset being matched is the actual modal split of residents of Singapore, calculated using ridership data from the 2010 Census of Population [21]. Using randomly generated coefficients does not compromise the validity of the model, because the input values for utility equations have arbitrary units of measurement (hours, dollars), and as such, they naturally require coefficients to become related. Mode bias coefficients also account for social values and other 
generally incalculable factors as discussed at length by Koppelman and Bhat [12].

Methods for modeling congestion are a key difference between the traditional four-step model and the proposed MTM. Traditional models rationalize congestion as an imbalance between trip demand and link capacity, and usually depend on equilibrating these two forces by reassigning trips within a feedback loop (McNally 2007). However, empirical evidence indicates that travelers continue to use heavily congested roads. Therefore, this understanding of congestion can fail to accurately represent reality, particularly due to induced demand. Instead, the MTM uses a common sense algorithm (Figure 4) based on causality, which utilizes the concept of congestion indices. A congestion index is a ratio that compares trip duration during peak travel times (morning and evening rush hours) with free-flowing travel (usually from 2-5am). For this MTM application, the base congestion indices were calculated using trips entered into TomTom MyDrive [22], a time-based route calculator that uses data sourced from real GPS devices. In the proposed algorithm, congestion indices are primarily modified by vehicle population, which is in turn modified by additional parameters such as road tolls, ride-sharing ratios, and total population. Congested travel is assumed for $50 \%$ of trips, with the congestion index averaged between values at the start and end of each trip [15].

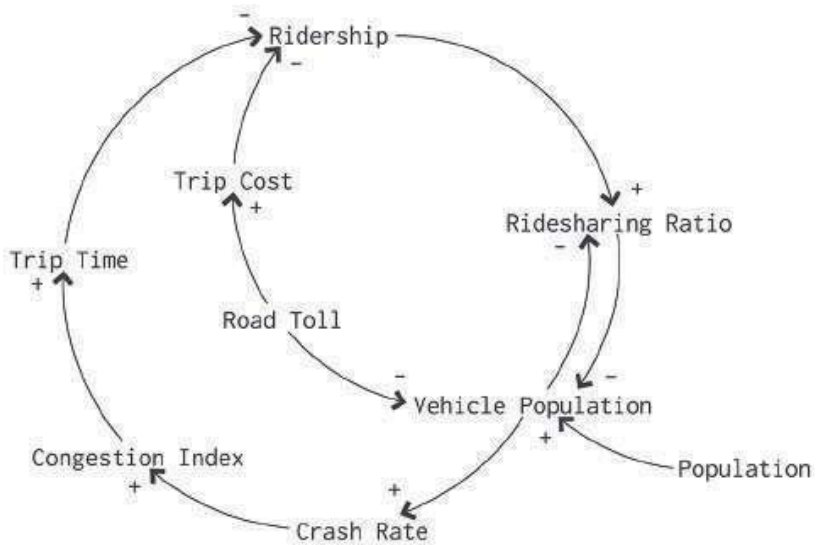

Figure 4. Causal loop diagram of the algorithm to calculate congestion indices for the MTM based on realistic, relevant factors.

Another key difference between the MTM and the four-step model is the speed with which the model can return results. Although four-step model practitioners have become reasonably accurate at predicting traffic demand and link capacity over the past six decades, the process is not optimal. Achieving a high degree of accuracy requires complex networks with long calculation times, and additional steps for advanced methods like time-of-day disaggregation and congestion feedback loops [3]. It is reasonable to conclude that although the four-step model is a powerful tool, it can be cumbersome and even restrictive. Comparing the architecture of the traditional four-step model with the MTM described in this paper reveals the MTM's clear advantages in terms of calculation time (Figure 5). To consider changes to the network, a four-step model must loop back to the trip distribution step, while changes in activity production must be recalculated from the trip generation step [16]. In comparison, once it has been initialized, the MTM can skip the costly trip generation and route assignment steps, giving the user immediate visual response, and a tactile experience.

\section{METHODS}

Implementing the MTM requires collecting an intensive quantity of site-specific data, including geospatial information for expressways, metro lines, bus routes, and bike paths. A geometric dataset capturing transportation infrastructure in Singapore was assembled in Rhinoceros $3 \mathrm{D}$, including data published by the Singapore Land Transport Authority regarding future infrastructure projects. Singapore is an ideal study area because within the last decade, they have built multiple new metro lines, producing several diverse data points for comparison. Singaporean institutions frequently publish high-quality data and are also conducting ongoing research into AVs, which indicates that the city will probably be an early adopter of robot car technology to enable further comparison $[11,20]$. The geometric data for existing and future infrastructure was organized chronologically into layers, and a Python script to cycle through the possible configurations while exporting the route data being produced from Grasshopper into JSON format.

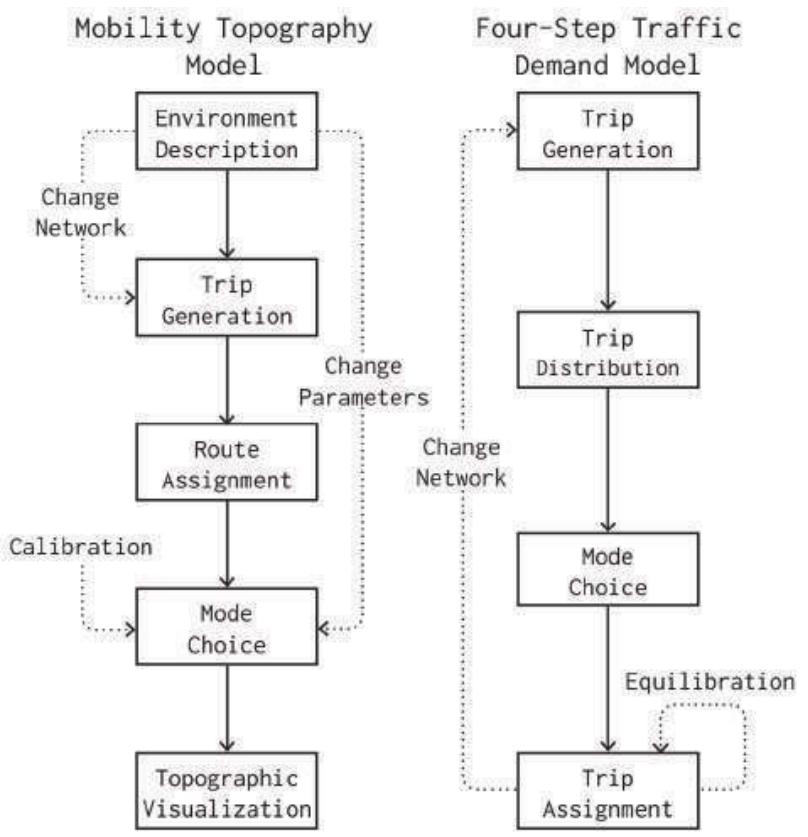

Figure 5. Flow chart comparing the inefficient architecture of the traditional FSM with the innovative architecture of the MTM designed to improve calculation speed and flexibility. 
However, options for reconfiguring infrastructure represent only a small percentage of the complete set of adjustable parameters available to the user. The quantity of possible scenarios that can be considered by modifying MTM parameters is endless. For example, vehicle speed can be increased to consider scenarios with $\mathrm{AVs}$ travelling at high speeds on dedicated expressways, or set to zero to simulate the complete removal of the expressway network. Highcapacity trip generating occupancies like stadiums can be strengthened to anticipate travel patterns for large events. There are also parameters available to consider travel time reliability to understand the possible implications of service disruptions on metro systems, or the effects of severe storms from climate change on bicycle use. The design of the MTM makes it flexible enough to quickly visualize diverse scenarios that traditional models may struggle to consider. Although the calculation method is similar to that of the four-step model it is not intended for same type of precise accounting, but instead for projecting broad trends and their consequences on the urban mobility landscape.

Implementing the following steps (trip generation and route assignment) is very similar to the four-step model. The data aggregated in Rhinoceros3D is processed in Grasshopper via algorithms written using a combination of native Grasshopper components and custom Python scripts (Figure 6). The shortest walk component developed by Piacentino [19] is also used extensively in the route assignment step to calculate optimal routes over expressway, metro, and bike path networks. In addition, a technique previously implemented by Cukier [5] for penalizing transfers between metro lines is applied for calculating metro routes.

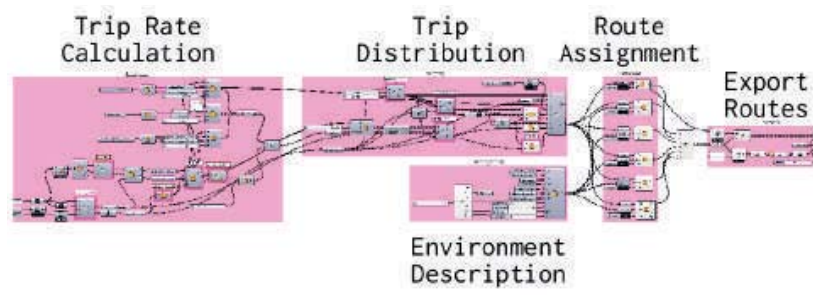

Figure 6. Screenshot of the Grasshopper3D script used to interpret the digitally described environment and to generate route data for subsequent mode choice calculations.

Route data was pre-calculated for every possible combination of a discrete number of infrastructural configurations, and exported in JSON format for use in the subsequent stages of the model. For the following steps of the MTM, the development environment was switched from Grasshopper3D to Processing, a popular Java development environment.

Route data based on Singapore's 2009 infrastructure and urban conditions was compared with ridership data from the 2010 census, and used to calibrate the model's mode utility coefficients using a GA adapted from a kernel developed by Turner [23]. With each generation of the GA, the time and cost impedance for every trip across every mode was evaluated and combined with a population of coefficients to output a set of mode utility values. Next, these values were converted to modal choice probabilities using the probability equation from Figure 2, and evaluated for fitness. An individual was considered fit if the calculated probable modal split aligned with actual modal split extracted from ridership statistics in the 2010 census for each analysis zone. After finding a solution below the threshold of $0.03 \%$, the GA stored the fittest individual and moved on to the next zone with a fresh population. Once routes were pre-calculated and optimal coefficients determined, these datasets were input to the Processing application designed to facilitate interaction with the MTM. At startup, time and cost values for each route are calculated by the application and stored in memory. Next, these route impedance values are combined in the calibrated MNL, and probability values are output. The most probable mode is determined for each trip start point and the color corresponding to that mode is assigned to a mesh vertex to construct the topographic mobility map visualization.

The application interface was designed to be simple and intuitive, while offering the user complete control over the entire range of mobility model parameters (Figure 7). Although the application is currently focused on Singapore, it can be adapted to model any urban environment. All of the graphical and numerical MTM output data presented in the following section was sourced from interaction with the application.

\section{RESULTS AND DISCUSSION}

This section is intended to demonstrate the process of analyzing MTM map and data outputs. It investigates the probable impact of AVs on mobility in Singapore with a series of three scenarios that could potentially occur over 30 years of technological and infrastructural evolution.

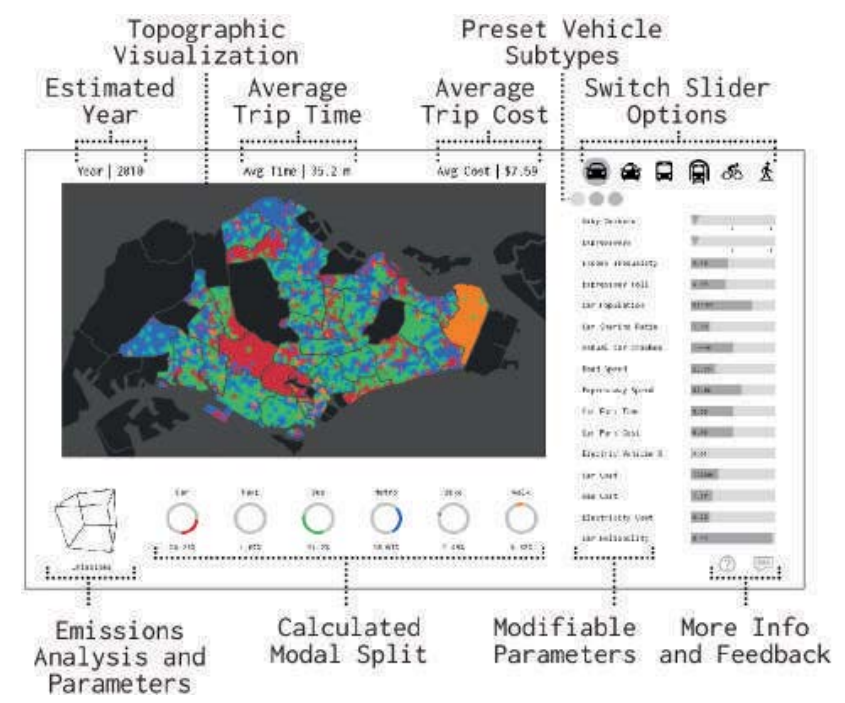

Figure 7. Screenshot of the interactive MTM interface complete with sliders for modifying parameters, a map viewport, and multiple sources of numerical data output. 


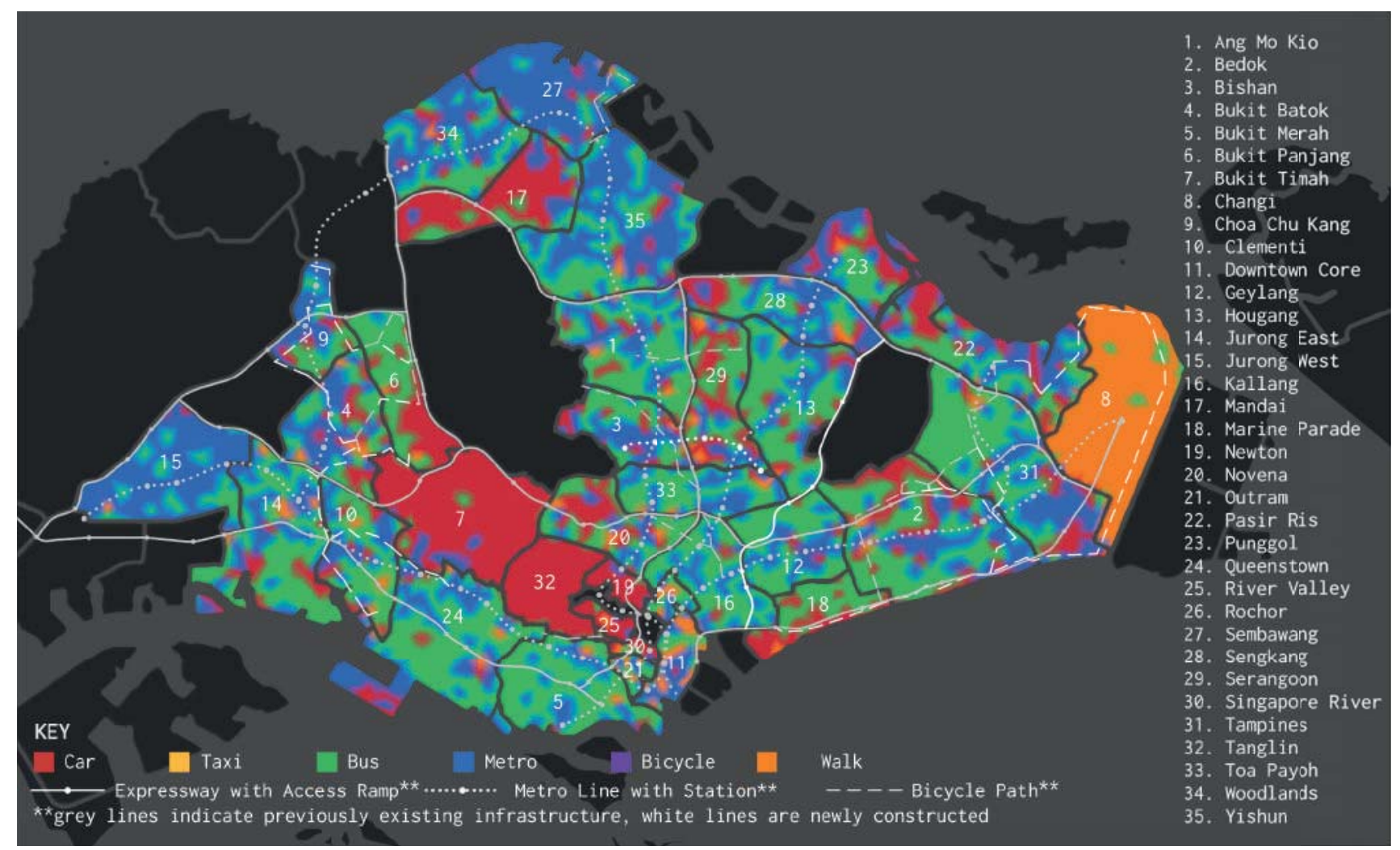

Figure 8. Probable modal choice visualized as a topographic map using data output by the MTM for scenario one (Singapore c. 2010). This mapping technique reveals the mobility layer of cities. Each place is understood as a product of local transportation habits and infrastructure.

- Scenario one: a snapshot of Singapore in 2009, based on residents' responses to the 2010 census.

- Scenario two: a projection of Singapore in 2025, with several major infrastructure projects completed and AVs in place of manually driven vehicles.

- Scenario three: a hypothetical Singapore in 2040, where $\mathrm{AVs}$ are present and the entire expressway system has been removed.

When examining the MTM output from scenario one at this holistic scale, several features of the existing mobility topography in Singapore become apparent (Figure 8). Notably, there is widespread reliance on bus travel despite frequent long wait times. Next, the residents of wealthy planning zones such as Mandai, Bukit Timah, and Tanglin are car dependent, while most other districts present a marbled transportation mix. The patterns of dominance apparent around metro lines and car expressways provide satisfactory evidence that the model has been properly formulated. The frequent dominance of walking over other modes visible in the Downtown Core and appearing elsewhere sporadically is a notable feature characteristic of walkable cities such as Singapore. Walking is dominant in Changi, a zone with a small population, low average income, and a large airport. Besides displaying the mobility topography map for Singapore in 2010, this image also introduces the graphic standards that will be used throughout this section. The subsequent diagrams illustrating MTM output display magnified, side-by-side comparisons of the three scenarios under analysis. The areas selected for detailed analysis demonstrate dramatic changes from modifying input parameters.

Scenario two assumes that road vehicles including cars, taxis, and even buses have become almost completely autonomous by 2025. Such a paradigm shift can be represented in the model by modifying parameters including parking time, vehicle speed, perceived cost, and crash rates. When AVs with piloted parking capabilities become prevalent, zero time is spent parking because passengers can travel door-to-door and leave the car to park itself [17]. Average road speeds will also increase slightly for shared streets, since autonomously managed intersections can be navigated without stopping, while expressway speeds may be faster because AVs can drive in platoon formation, reducing both energy consumption and congestion [7, 10]. These reductions in trip time for car journeys increase the probability of car travel.

The concept of perceived cost describes the monetary equivalent for stress caused by the act of driving, and varies according to road conditions and the purpose of the trip [14]. As people become more comfortable in AVs, driver stress will be reduced and time can be spent performing tasks other than driving, changing existing perceptions of the value of travel time. The potential downside of 
perceived cost reduction is radically expanded urban sprawl, since travellers would be willing to spend more time commuting if their cars did the work of driving.

$\mathrm{A}$ benefit of $\mathrm{AVs}$ is that they remove the risk of human error from the roads, and could reduce the frequency of car crashes by $90 \%$ [8]. This is represented in the model by reducing the crash cost carried by travellers. Crash cost is a monetary value assigned per kilometer of travel to cover the internal costs of a crash including property damage, medical expenses, lost wages, etc., based on the probable severity of an accident.

A fleet of autonomous taxis could also eliminate the danger of drunk drivers because they would be readily available and cheaper to operate than traditional taxis with drivers. The associated increase in taxi modal share would expand the taxi population and reduce wait times outside of the city center. Research suggests that shared autonomous taxis could reduce the population of privately owned vehicles within urban environments at a ratio of ten fewer cars for each taxi added [9]. The MTM translates this decrease in congestion over surface routes as growth in the attractiveness of car, taxi, and bus travel.

The transportation infrastructure in scenario two has also been updated to include projects that are likely to be completed by 2025, including expanded metro lines, expressways, and bike paths. For this scenario, the total population of Singapore was increased in line with predictions from the Population White Paper, causing an increase in congestion experienced across every mode [18].

\begin{tabular}{|c|c|c|c|c|c|c|}
\hline \multirow{2}{*}{ Year } & \multicolumn{6}{|c|}{ Calculated Modal Split } \\
\cline { 2 - 7 } & Car & Taxi & Bus & Metro & Bike & Walk \\
\hline 2010 & $24.3 \%$ & $1.7 \%$ & $34.2 \%$ & $30.7 \%$ & $2.5 \%$ & $6.6 \%$ \\
\hline 2025 & $22.9 \%$ & $18.4 \%$ & $28.1 \%$ & $23.7 \%$ & $2.0 \%$ & $4.9 \%$ \\
\hline 2040 & $22.5 \%$ & $8.1 \%$ & $25.0 \%$ & $33.0 \%$ & $6.9 \%$ & $4.5 \%$ \\
\hline
\end{tabular}

Table 1. Modal splits for the three different transportation scenarios analyzed in this section.

\begin{tabular}{|c|c|c|c|}
\hline Year & $\begin{array}{c}\text { Average } \\
\text { Trip Time } \\
\text { (minutes) }\end{array}$ & $\begin{array}{c}\text { Average } \\
\text { Trip Cost } \\
\text { (SGD) }\end{array}$ & $\begin{array}{c}\text { Annual } \\
\text { Transport } \\
\text { Emissions } \\
\text { (MT CO } \mathbf{2}_{\mathbf{2}} \text { ) }\end{array}$ \\
\hline 2010 & 35.2 & $\$ 7.59$ & 8.1 \\
\hline 2025 & 28.2 & $\$ 9.65$ & 7.8 \\
\hline 2040 & 31.4 & $\$ 6.16$ & 5.5 \\
\hline
\end{tabular}

Table 2. Average trip time and cost comparison, along with annual emissions to evaluate the impacts of each scenario.

The third scenario is a more distant projection of Singapore as it might exist in 2040, where the main assumption is that the entire network of restricted-access expressways has been removed. This significant change is simulated by reducing vehicle speed on expressways to zero, forcing the model to consider only direct routes for cars and taxis. Other parameters changed between the second and third scenarios include further incremental improvement in wait times, and slightly reduced vehicle speed over local roads due to higher congestion. Metro and bike infrastructure were also updated to include more bike rental points and reduce bike park times, assuming that more racks will be available. The total population of Singapore is again increased. The resulting output data from these three scenarios is summarized in Tables 1 and 2 above.

To demonstrate the mobility topography analysis process, the first area of focus is the wealthy and car-dependent planning zone Bukit Timah, and the adjacent zones Bukit Batok, Bukit Panjang, Clementi, and Jurong East (Figure 9). The magnified map of 2025 suggests that residents of Bukit Timah would prefer private AVs over mass public transit despite receiving a metro line. Moreover, car dominance in Bukit Timah will not be superseded by cheaper and more readily available taxis, a conclusion that makes sense in the context of Singapore, where car ownership is a status symbol. Meanwhile, residents who previously relied upon mass transit or walking in the suburban, middle income zones Bukit Batok, Clementi, and Jurong East may switch to autonomous cars and taxis for their speed and convenience.
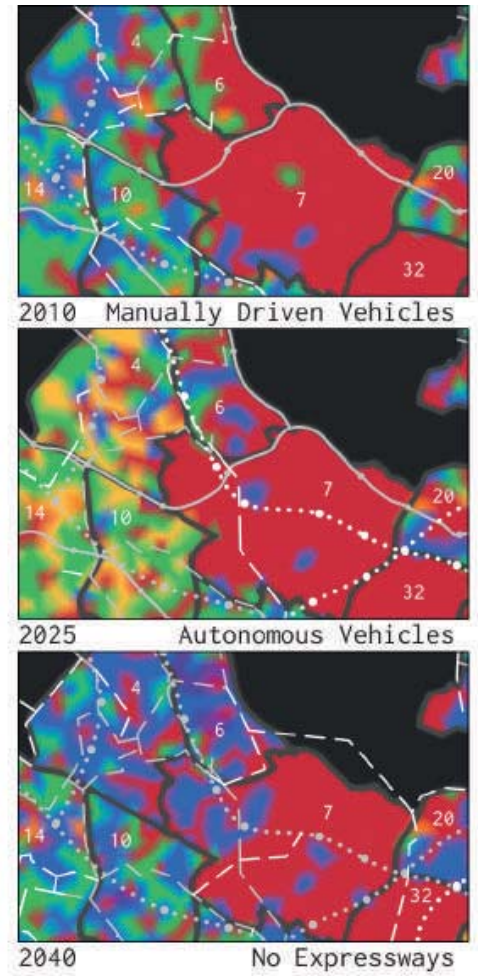

Figure 9. Magnified MTM output for three scenarios focused on luxurious residential district Bukit Timah. 

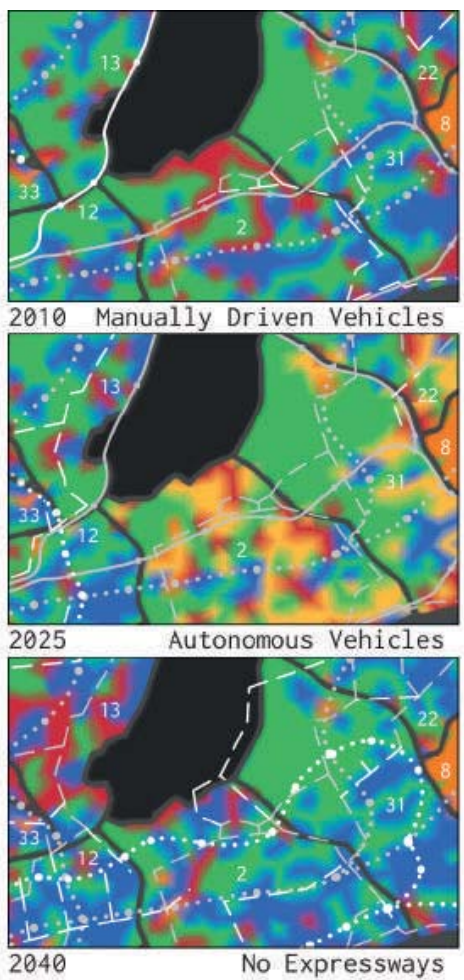

Figure 10. Magnified MTM output for three scenarios focused on dense, affordable residential zones Bedok and Tampines.

However, when expressways are removed in 2040 for scenario three, the MTM suggests that a significant percentage of Bukit Timah's population would finally utilize the local public transit infrastructure. Personal vehicle use in the adjacent zones is also projected to decrease. Notably, the expanded bike path networks of 2040 appear to result in pockets within Bukit Panjang where bikes are the preferred mode of personal transport.

The second area for comparison analysis is focused on the regional center of Tampines, and the adjacent zones Bedok, Hougang, and Pasir Ris (Figure 10). These are densely populated, medium income residential zones where present day occupants rely on a mixture of car, bus, and metro transport for their commutes. When AVs are introduced into this environment, the MTM predicts that much of Pasir Ris and large swaths of Bedok and Tampines would switch to autonomous cars or taxis, despite the $23.8 \%$ increase in average cost per trip. Reduced dependence on slow bus transport probably accounts for much of the $19.9 \%$ reduction in average travel time predicted by the model. It is worth noting that the substantial increase in the quantity of vehicles present may increase congestion and degrade the quality of life for residents of these areas.

However, without expressways the calculated probability for residents of Tampines, Bedok, and Pasir Ris to travel by car drops dramatically by 2040 . The model predicts they would switch to more environmentally sustainable bus or metro transport, while in Hougang, the reduction in vehicle population and congestion is projected to cause a slight increase in car use. The general shift towards mass transit predicted by the MTM comes with an $11.3 \%$ increase in average travel time, while the average cost per trip drops by an astonishing $31.1 \%$. The implication of this analysis is that expressways and tolls perpetuate a two-tier transportation system. Wealthier travellers can afford fast personal mobility, while the inhabitants of low and middle income areas are more likely to avoid travelling by car or taxi because of the relatively high cost. Without expressways dividing the social and urban fabrics, the model concludes that mobility would be more democratic and substantially cheaper. Some of the potential benefits in this scenario would be greater social cohesion and growing average personal wealth.
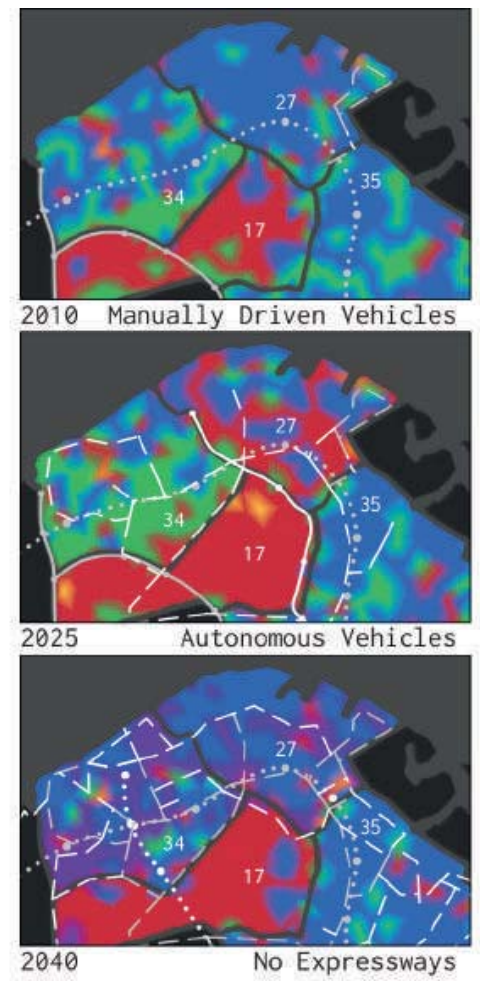

Figure 11. MTM output for three scenarios focused on emerging employment clusters in zones Woodlands and Sembawang.

The emerging regional center at Woodlands and the adjacent zones Sembaweng, Mandai, and Yishun form the third area of focus for detailed analysis (Figure 11). A comparison between the MTM output for 2010 and 2025 reveals that many residents of Sembaweng and Mandai are projected to shift towards car dependence upon completion of the North-South expressway in 2020. The model predicts that residents of Woodlands would prefer to travel by bus rather than by metro in this scenario. This phenomenon is a result of the growing use of autonomous taxis seen in previous analyses, which will reduce the number of vehicles in Singapore. Decreasing congestion on surface routes is projected to lead to a corresponding increase of 
bus use in Woodlands.

For the third scenario, the previous increase in car use would be completely negated by the elimination of highspeed expressways. In fact, the MTM concludes that the expanded bike path network will lead to a significant percentage of residents in Sembaweng and Woodlands commuting by bike. This implies that residents might work nearby and not travel to the Downtown Core, thereby changing the wasteful commuting pattern prevalent in cities around the world. The MTM analysis concludes that by combining employment growth in the Woodlands regional center with expressway-free transportation, Singapore could transform into an example of a sustainable mobility city.

\section{FUTURE WORK}

Although this sample implementation in Singapore includes a substantial amount of data collected from site-specific sources such as Singapore's Land Transport Authority, the MTM could also function with readily available data from Google. Acquiring a reasonable amount of data over Google's API and compiling mobility topography maps would be fast and relatively straightforward for many cities. Differences and similarities in the algorithms and coefficients across diverse socio-cultural regions may become apparent to a trained neural network, and broader theories of urban transportation could eventually be developed. A faster model could also be applied to the task of charting transportation lines to optimize a particular parameter, such as metro ridership or travel time.

An issue with the MTM is its simplistic understanding of multimodal travel. The model does not consider the breakdown of individual trips, instead concentrating on the more general concept of ridership. By definition, a multimodal trip counts towards two or more rides, implying that by focusing on ridership the proposed model considers intensity of use and not actual travel patterns. Future iterations of the model could implement more complete understandings of multimodality by introducing combined modes to the logit model, such as "car and metro" or "bus and bike." Unfortunately, adding additional dimensions increases calculation time exponentially. This could also compromise the accuracy of the coefficients, since GA performance degrades with higher dimensional problems. The model may also benefit from expanding the quantity of routes available for each trip to dynamically redistribute trips away from congested routes.

In the seven years that have elapsed since the collection of Singapore's 2010 census data, the city has added $7 \mathrm{~km}$ of expressways, $46.8 \mathrm{~km}$ of metro lines, and $124.5 \mathrm{~km}$ of bike paths. This rapid pace of transportation infrastructure development presents an opportunity to evaluate the accuracy of the MTM. Singapore's evolving infrastructure will have impacted transportation patterns over the last seven years. If a survey of the present day transportation profile for a significant percentage of Singaporeans agreed with the predictions of the MTM, it would confirm the usefulness of the model as a projection tool. At minimum a survey would offer more data for comparison. The interactive MTM application is hosted online, presently generating a database of feedback for further analysis.

\section{CONCLUSION}

The intent of this project was to construct a predictive model that would complement the transportation planners existing toolkit. Although the accuracy of this model is unproven, it may still offer valuable perspective to urban planners. The linear structure of the MTM enables faster, more tactile visualization than can be achieved with traditional modeling techniques. The simplified congestion model implemented by the MTM also allows it to simulate a broad range of scenarios without experiencing capacity overloads and "breaking," as a traditional four-step model would. In theory, with further advancement this model could be used to generate optimal solutions to problems that escape the threshold for understanding complexity of the average human urban planner.

At present this project is best described as a mathematical probability model that visualizes the effects of changing variables over several layered geometric networks. It is still a speculative project without empirical evidence to validate its predictions. However, even if it remains merely an intermediary between the deductive abilities of the human imagination and the unimaginable complexity of an urban transportation system, it is still a potentially valuable tool for planners to consult during the decision-making process.

\section{REFERENCES}

1. Aschwanden, Gideon. 2012. "Agent-Based Social Pedestrian Simulation for the Validation of Urban Planning Recommendations.” Sigradi, 332-36.

2. Chen, Liang. 2012. "Agent-Based Modeling in Urban and Architectural Research: A Brief Literature Review." Frontiers of Architectural Research, 1: 166-77.

3. Chen, T. Donna, Kara M. Kockelman and Yong Zhao. 2015. "What Matters Most in Demand Model Specifications: A Comparison of Outputs." Journal of the Transportation Research Forum, 52 (1): 71-89.

4. Chimba, Deo, Daniel Emaasit and Boniphace Kutela. Oct. 2012. "Integrating Origin Destination Survey and Stochastic User Equilibrium: A Case Study for Route Relocation." Journal of Transportation Technologies, 2 (2): 297-304.

5. Cukier, Jerome. 2013. "Interactive Map of the Subway." Communicating with Data, Jan. 13. http://www.jeromecukier.net/blog/ 2013/01/14/interactive-map-of-the-subway/.

6. Diappi, Lidia, Paola Bolchim, and Massimo Buscema. 2004. "Improved Understanding of Urban Sprawl Using Neural Networks." In Recent Advances in Design \& 
Support Systems in Architecture and Urban Planning, $33-49$.

7. Dresner, Kurt and Peter Stone. 2008. "A Multiagent Approach to Autonomous Intersection Management." Journal of Artificial Intelligence Research, 31: 591-656.

8. Fagnant, Daniel and Kara M. Kockelman. 2015. "Preparing a Nation for Autonomous Vehicles: Opportunities, Barriers and Policy Recommendations for Capitalizing on Self-Driven Vehicles." Transportation Research Part A, 77: 167-181.

9. Fagnant, Daniel and Kara M. Kockelman. 2016. "Dynamic Ride-Sharing and Optimal Fleet Sizing for a System of Shared Autonomous Vehicles." Transportation. Accepted, Oct. 2015.

10. Fernandes, Pedro and Urbano Nunes. Mar. 2012. "Platooning With IVC-Enabled Autonomous Vehicles: Strategies to Mitigate Communication Delays, Improve Safety and Traffic Flow." IEEE Transactions on Intelligent Transportation Systems, 13 (1): 91-106.

11. Kheong, Tan Cheon and Tham Kwang Sheun. 2014."Autonomous Vehicles, Next Stop: Singapore." JOURNEYS (12): 5-11.

12. Koppelman, Frank S. and Chandra Bhat. 2006. A Self Instructing Course in Mode Choice Modeling. Multinomial and Nested Logit Models. U.S. Department of Transportation.

13.Lee, Kwang Sub, Jin Ki Eom and Dae-Seop Moon. 2014."Applications of TRANSMIS in Transportation: A Literature Review." Procedia Computer Science, 32: 769-77.

14.Litman, Todd. 2002. "The Costs of Automobile Dependency and the Benefits of Balanced Transportation." Victoria Transport Policy Institute.
15.Litman, Todd and Eric Doherty. Aug. 2013. "Travel Time Costs." In Transportation Cost and Benefit Analysis II. Victoria Transport Policy Institute.

16. McNally, Michael G. 2007. "The Four Step Model.” In Handbook of Transport Modeling. Emerald Group Publishing Limited.

17. Meier-Burkert, Friederike. 2014. "Piloted Parking." Audi Urban Future Initiative. http://audi-urban-futureinitiative.com/blog/piloted-parking-future-mobility.

18. National Population and Talent Division. Jan. 2013. Population White Paper: A Sustainable Population for a Dynamic Singapore. Singapore: Prime Minister's Office.

19. Piacentino, Giulio. May 2011. Shortest Walk Gh. Grasshopper3D. McNeel Europe. http://www.food4rhino.com/project/shortestwalkgh?etx.

20. Rodoulis, Stelios. 2014. "The Impact of Autonomous Vehicles on Cities." JOURNEYS (12): 12-20.

21. SINGSTAT. 2010. Census of Population 2010 Statistical Release 3: Geographic Distribution and Transport. Singapore: Department of Statistics.

22. TomTom MyDrive. 2016. TomTom International BV. https://mydrive.tomtom.com/.

23. Turner, Alasdair. 2009. Genetic Algorithm. Processing. http://www.openprocessing.org/sketch/3101.

24.Zhong, Ming, Pawan Lingras, Will Blades and John Douglas Hunt. 2008. "Evolving Parameters of Logit Models Using Genetic Algorithms.” In Canadian Transportation Research Forum.

25.Zhou, Bin (Brenda), Kara M. Kockelman and Jason D. Lemp. 2009. "Transportation and Land Use Policy Analysis Using Integrated Transport and Gravity-Based Land Use Models." Transportation Research Record, 2133: $123-32$ 